\title{
Real-Space Observation of Surface States on Sil111) 7×7 with the Tunneling Microscope
}

\section{Citation}

Becker, R. S., J. A. Golovchenko, D. R. Hamann, and B. S. Swartzentruber. 1985. Real-Space Observation of Surface States on Si(111) 7×7 with the Tunneling Microscope. Physical Review Letters 55, no. 19: 2032-2036. doi:10.1103/physrevlett.55.2032.

\section{Published Version}

doi:10.1103/PhysRevLett.55.2032

\section{Permanent link}

http://nrs.harvard.edu/urn-3:HUL.InstRepos:29407043

\section{Terms of Use}

This article was downloaded from Harvard University's DASH repository, and is made available under the terms and conditions applicable to Other Posted Material, as set forth at http:// nrs.harvard.edu/urn-3:HUL.InstRepos:dash.current.terms-of-use\#LAA

\section{Share Your Story}

The Harvard community has made this article openly available.

Please share how this access benefits you. Submit a story.

\section{Accessibility}




\title{
Real-Space Observation of Surface States on Si(111) $7 \times 7$ with the Tunneling Microscope
}

\author{
R. S. Becker, J. A. Golovchenko, D. R. Hamann, and B. S. Swartzentruber \\ AT\&T Bell Laboratories, Murray Hill, New Jersey 07974
}

(Received 25 July 1985)

\begin{abstract}
We have observed surface states indigenous to the $\mathrm{Si}(111) 7 \times 7$ superlattice with the tunneling microscope. The data show the states' energies and strengths to be strongly dependent on position in the unit mesh. Implications of this for structural models of the $7 \times 7$ superlattice are discussed.
\end{abstract}

PACS number: $68.20 .+\mathrm{t}$

The detailed electronic structure of the surface of semiconductors has attracted considerable interest in condensed-matter physics. Over the years, many techniques yielding complementary information have been brought to bear on this question. For example, photoemission spectroscopy ${ }^{1}$ and inverse photoemission spectroscopy ${ }^{2}$ give precise energy information on occupied and unoccupied states, respectively, but unfortunately average over a minimum of $10^{11}$ atomic sites. Techniques such as low-energy electron diffraction (LEED) ${ }^{3}$ and atom and ion scattering ${ }^{4}$ are capable of structural information, but analysis of their results depends on a detailed model of the surface. The recent advent of the tunneling microscope ${ }^{5}$ promises a tool capable of not only precise electronic detail but local probing of the surface on a size scale of a few angstroms.

In this Letter, we present data showing, for the first time, the lateral localization of surface states characteristic of the $\mathrm{Si}(111) 7 \times 7$ superlattice, and correlate them with the different substructures of the two halves of the unit mesh.

The sample was a boron-doped, $0.05-\Omega-\mathrm{cm} \mathrm{Si(111)}$ wafer, Syton polished to a mirror finish. Before characterization by the tunneling microscope, the $\mathrm{Si}$ wafer was oriented by Laue $\mathrm{x}$-ray diffraction, argon-ion sputter cleaned, and annealed at $900^{\circ} \mathrm{C}$ in ultrahigh vacuum. Examination with LEED showed a sharp diffraction pattern characteristic of well-reconstructed $\mathrm{Si}(111) 7 \times 7$. After approximately a 10 -h wait for thermal drifts to decay to acceptable levels, both topographic images and tunneling-field-emission spectroscopy data were obtained.

The operation of the tunneling microscope in its topographic imaging mode has been described by various authors ${ }^{5,6}$ elsewhere and will not be discussed in this Letter. In the microscope's tunneling-field-emission spectroscopy mode, we superimpose a small $(0.1 \mathrm{~V})$ ac signal at $4.5 \mathrm{kHz}$ on the dc bias of the vacuum gap, and employ a lock-in amplifier for detection of the corresponding in-phase ac component in the tunneling current. The microscope's feedback loop, which has a frequency response much less than the frequency of the ac dither signal, maintains a constant dc tunnel current at about $1 \mathrm{nA}$. With the tip poised over the region of interest, the $\mathrm{dc}$ bias is ramped from the vacuum-tunneling through field-emission regimes to give a spectrum of conductance $(d I / d V)$ vs $V_{\text {bias }}$ in a few seconds. Topographic imaging before and after shows the tip to maintain its lateral position to within 1 $\AA$ during a spectroscopy run.

Figure 1(a) shows a topographic image of the region of the silicon surface that was used in acquiring tunneling-field-emission spectra. The data are displayed in a gray-scale format, where light corresponds to raised areas, and dark to depressed areas. This tunneling image was acquired at a bias voltage of $2.0 \mathrm{~V}$ with tunneling from tip to sample, chosen for reasons of contrast and microscope operation. The $\mathrm{Si}(111) 7 \times 7$ unit mesh is diamond shaped, as indicated in the figure, and consists of four deep depressions (corner holes) surrounding back-to-back equilateral triangles. Each triangular subunit contains six protrusions which are suggested to be adatoms. ${ }^{5}$ The triangular subunit with vertices pointing in $\langle 2 \overline{11}\rangle$ directions is labeled $A$, while the triangular subunit with vertices pointing in $\langle\overline{2} 11\rangle$ directions is labeled $B$. The $\mathrm{X}$ and the $\mathrm{o}$ designate the centers of triangles $\mathrm{A}$ and $\mathrm{B}$, respectively. Figure 1 (b) shows a simultaneously acquired conductance image, also displayed in gray scale, with light indicating large signal and dark denoting lesser signal. One immediately sees that the A triangles appear light and the B triangles dark. The protrusions inherent in each triangle are imaged as dark spaces, while the point defects apparent in Fig. 1(a) appear as either a loss of signal (black), or increased conductance (white) for vacancies. Clearly, this mapping of conductance at a fixed bias voltage of $2.0 \mathrm{~V}$ demonstrates the localization of the electronic density of states to fixed points on the superlattice as small as a few angstroms in extent.

A conductance spectrum versus $V_{\text {bias }}$ is shown for both triangles $\mathrm{A}$ and $\mathrm{B}$ of the $\mathrm{Si}(111) 7 \times 7$ unit mesh in Fig. 2, along with plots of the tip trajectory at constant tunnel current. These data were taken at the points $x$ and $o$ shown in Fig. 1 at several defect-free triangles of both varieties. The large peaks beginning at $5-\mathrm{V}$ bias and extending up through the field-emission region 


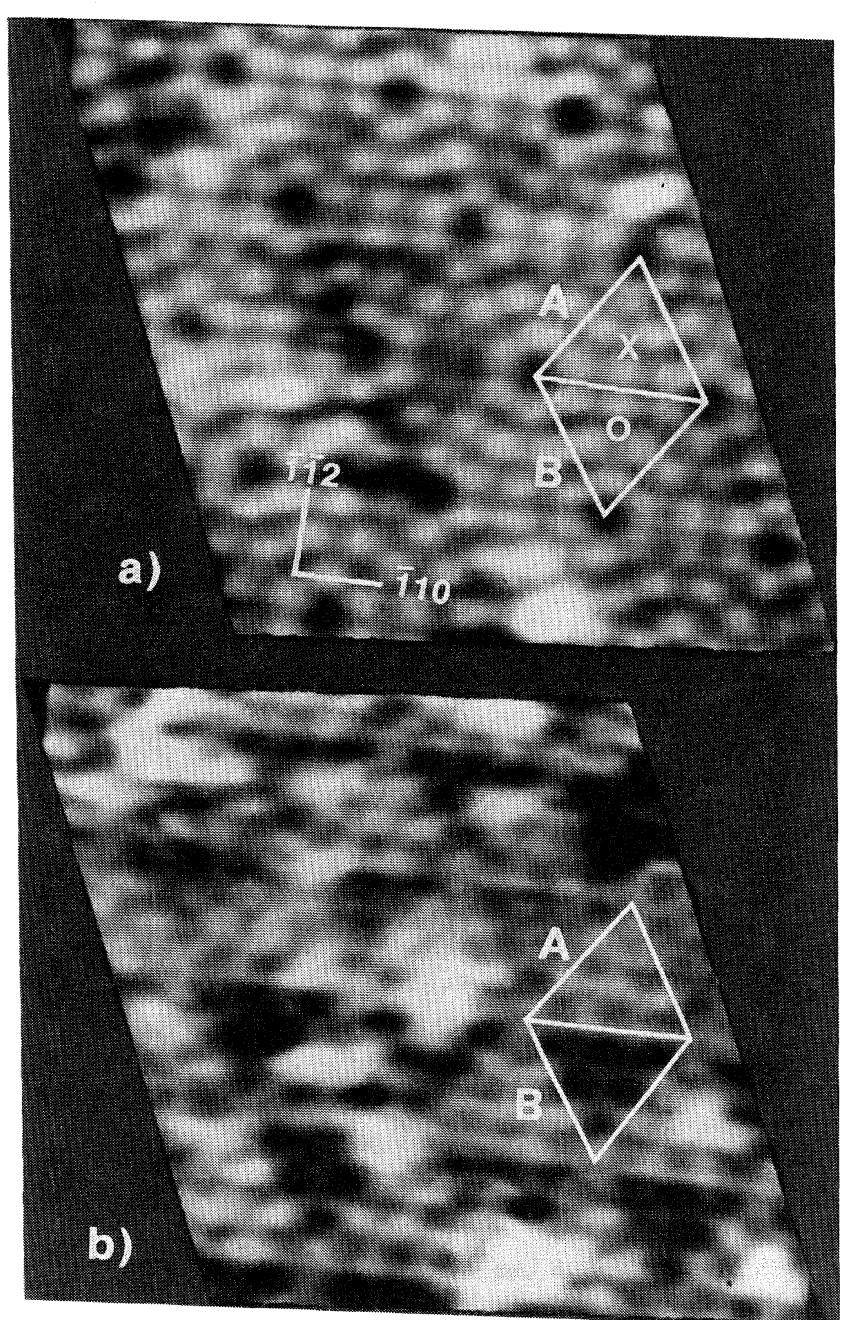

FIG. 1. (a) Gray-scale tunneling image of the $\mathrm{Si}(111) 7 \times 7$ surface used for the tunneling-field-emission studies. Light areas are high, while dark areas are low, with a total range of $1 \AA$. (b) Simultaneous conductance image with light representing increased signal and dark representing decreased signal (arbitrary units). The $7 \times 7$ unit mesh is depicted as a rhombus built up from two equilateral triangles. The surface orientation is as shown, with the A and B designating triangular subunits with vertices pointing in $\langle 2 \overline{11}\rangle$ and $\langle\overline{2} 11\rangle$ directions, respectively. The points $\mathrm{x}$ and $o$ indicate the centers of the subunits.

are characteristic of electron standing waves whose details are described elsewhere. ${ }^{7}$ Here the standing-wave theory, applied to the conductance data, conveniently serves to determine the location of the vacuum, shown in the figure as a dashed vertical line at $V_{\text {bias }}=4.4 \mathrm{~V}$. We prefer to reference energy positions from the vacuum rather than the Fermi level since the location of the latter is somewhat uncertain because of unknown contributions from band bending and Schottky bar-

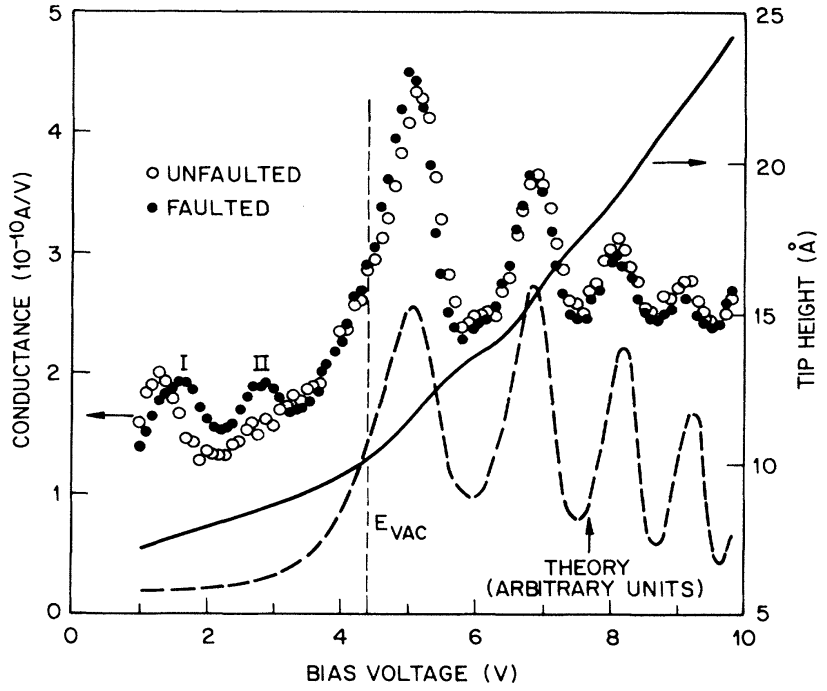

FIG. 2. Conductance spectra vs $V_{\text {bias }}$ for the $\mathrm{Si}(111) 7 \times 7$ surface. The filled circles and open circles denote data taken with the tip centered over faulted and unfaulted triangular subunits, respectively. The solid line shows the vacuum gap vs $V_{\text {bias }}$ during the conductance measurements. The vacuum level is indicated, with the dashed curve showing the theoretical standing-wave conductance oscillations for the observed dependence of vacuum gap on $V_{\text {bias }}$.

riers.

The spectruin in Fig. 2 plotted with closed circles shows two peaks below the vacuum which we have found to be characteristic of reconstructed $\mathrm{Si}(111)$ in general. These data are the result of measurements centered over type-A triangular subunits. The data indicated by open circles in Fig. 2 were acquired in an identical manner, with the tip, however, positioned over type-B triangular subunits. While virtually identical to the type-A spectrum in the field-emission regime, it is immediately seen that one of the peaks below the vacuum (labeled II, at $-1.6 \mathrm{~V}$ ) has nearly disappeared, while the peak at $-2.8 \mathrm{~V}$ (labeled I) has shifted down in energy some $0.3 \mathrm{~V}$.

Early on, tunneling microscopy by Binnig et al. ${ }^{5}$ showed the $\mathrm{Si}(111) 7 \times 7$ unit mesh to be asymmetric across the short diagonal. This result has been confirmed ${ }^{8}$ and quantified to show that the bases of the triangular subunits pointing in $\langle\overline{2} 11\rangle$ directions (typeB) are raised, while those pointing in $\langle 2 \overline{11}\rangle$ directions (type-A) are lowered, with a height difference of approximately $0.2 \mathrm{~A}$. Furthermore, the data supported a dimer adatom stacking-fault (DAS) model proposed by Takayanagi et $a l^{9}$ In this model the outermost complete double layer in the unit mesh consists of two triangular subunits that are respectively faulted and unfaulted with respect to the substrate. This determination of sample orientation forces the lowered type-A subunits to be faulted, while the raised type-B 


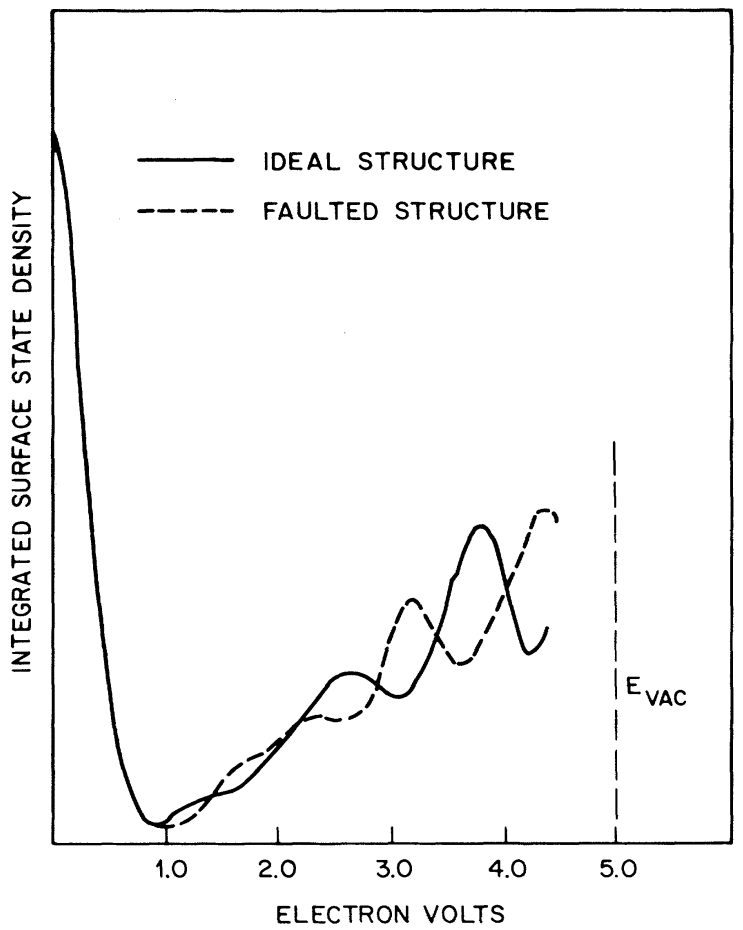

FIG. 3. Theoretical density of states vs energy for faulted and unfaulted $\mathrm{Si}(111)$ plane slabs. The curves were calculated by the linear-augmented-plane-wave density-functional method described in the text.

triangles are unfaulted. Hence, we assign the second peak (II) in the data in Fig. 2 to electronic states inherent in the faulted structure of the unit mesh and its absence in the type-B triangles to detailed differences in electronic structure brought about by the stacking fault. It seems likely that the shift in position in the peak at $-2.8 \mathrm{~V}$ (I) is also tied to the alternate faulted-unfaulted stacking. We note in passing that the first peak (I) in Fig. 2 can be identified in the inverse photoemission data of Himpsel, and Fauster. ${ }^{10}$ They go on to show that this state is not present on $\mathrm{Si}(100) 2 \times 1$, further tying its existence to the $7 \times 7$ superstructure.

A preliminary calculation testing whether the presence of a surface stacking fault can significantly affect the density of states between the Fermi level and the vacuum has been done and is shown in Fig. 3. The calculation is based on a twelve-layer slab with use of linear augmented plane waves within the selfconsistent local-density-functional formalism. ${ }^{11}$ This method is well suited to treating tunneling-microscope problems because the decaying wave-function tails in the vacuum are represented by accurate numerical basis functions. ${ }^{12}$ The surface density of states is obtained by integration over the decaying wave-function tails from a plane $1.3 \AA$ above the surface out to infini- ty and over the entire Brillouin zone. We feel that the presence of the adatoms and dislocations in the $7 \times 7$ structure provides enough scattering to thoroughly fold the $1 \times 1$ zone and average over surface bandstructure effects. While too simplistic to allow rigorous connection of the peaks in the conductance data to the peaks in the calculated density of states, it demonstrates that large effects on the density of states in the vacuum-tunneling regime can be traced to structural changes in the outermost double layer. Indeed, it has been shown ${ }^{13}$ that a bulk stacking fault produces localized electronic states, and it is not unreasonable to expect that they could hybridize with surface-induced states.

In conclusion, we have observed surface states indigenous to the $\mathrm{Si}(111) 7 \times 7$ superstructure with the tunneling microscope using vacuum-tunneling-fieldemission spectroscopy. We further show that their positions and strengths depend strongly on position in the unit mesh, and we have gone on to identify them with structural differences in the two sides of the unit mesh.

We would like to acknowledge helpful discussions with E. G. McRae of AT\&T Bell Laboratories.

${ }^{1}$ B. Feuerbacher, B. Fitton, and R. F. Willis, Photoemission and the Electronic Structure of Surfaces (Wiley, New York, 1978).

${ }^{2}$ N. V. Smith, in Proceedings of the Sixth Interdisciplinary Surface Science Conference, University of Warwick (to be published); V. Dose, Progr. Surf. Sci. 13, 225 (1983); H. Scheidt, Fortschr. Phys. 31, 357 (1983); Th. Fauster and F. J. Himpsel, J. Vac. Sci. Technol. A 1, 1111 (1983).

${ }^{3}$ E. G. McRae, Rev. Mod. Phys. 51, 541 (1979).

${ }^{4}$ M. J. Cardillo, G. E. Becker, D. R. Hamann, J. A. Serri, L. Whitman, and L. F. Mattheiss, Phys. Rev. B 28, 494 (1983); M. J. Cardillo, Phys. Rev. B 23, 4279 (1981); M. Aono, R. Souda, C. Oshima, and Y. Ishizawa, J. Vac. Sci. Technol. A 3, 1514 (1985).

${ }^{5}$ G. Binnig, H. Rohrer, Ch. Gerber, and E. Weibel, Phys. Rev. Lett. 50, 120 (1983).

${ }^{6}$ R. S. Becker, J. A. Golovchenko, and B. S. Swartzentruber, Phys. Rev. Lett. 54, 2678 (1985).

${ }^{7}$ R. S. Becker, J. A. Golovchenko, and B. S. Swartzentruber, Phys. Rev. Lett. 55, 987 (1985).

${ }^{8}$ R. S. Becker, J. A. Golovchenko, E. G. McRae, and B. S. Swartzentruber, preceding Letter [Phys. Rev. Lett. 55, 2028 (1985)].

${ }^{9} \mathrm{~K}$. Takayanagi, Y. Tanishiro, M. Takahashi, and S. Takahashi, J. Vac. Sci. Technol. A 3, 1502 (1985).

${ }^{10}$ F. J. Himpsel and Th. Fauster, J. Vac. Sci. Technol. A 2 , 815 (1984).

${ }^{11}$ O. K. Anderson, Phys. Rev. B 12, 3060 (1975); O. Jepsen, J. Madsen, and O. K. Andersen, Phys. Rev. B 18, 605 (1978); D. R. Hamann, Phys. Rev. Lett. 46, 1227 (1981).

12J. Tersoff and D. R. Hamann, Phys. Rev. Lett. 50, 1998 (1983).

${ }^{13}$ L. F. Mattheiss and J. R. Patel, Phys. Rev. B 23, 5384 (1981). 


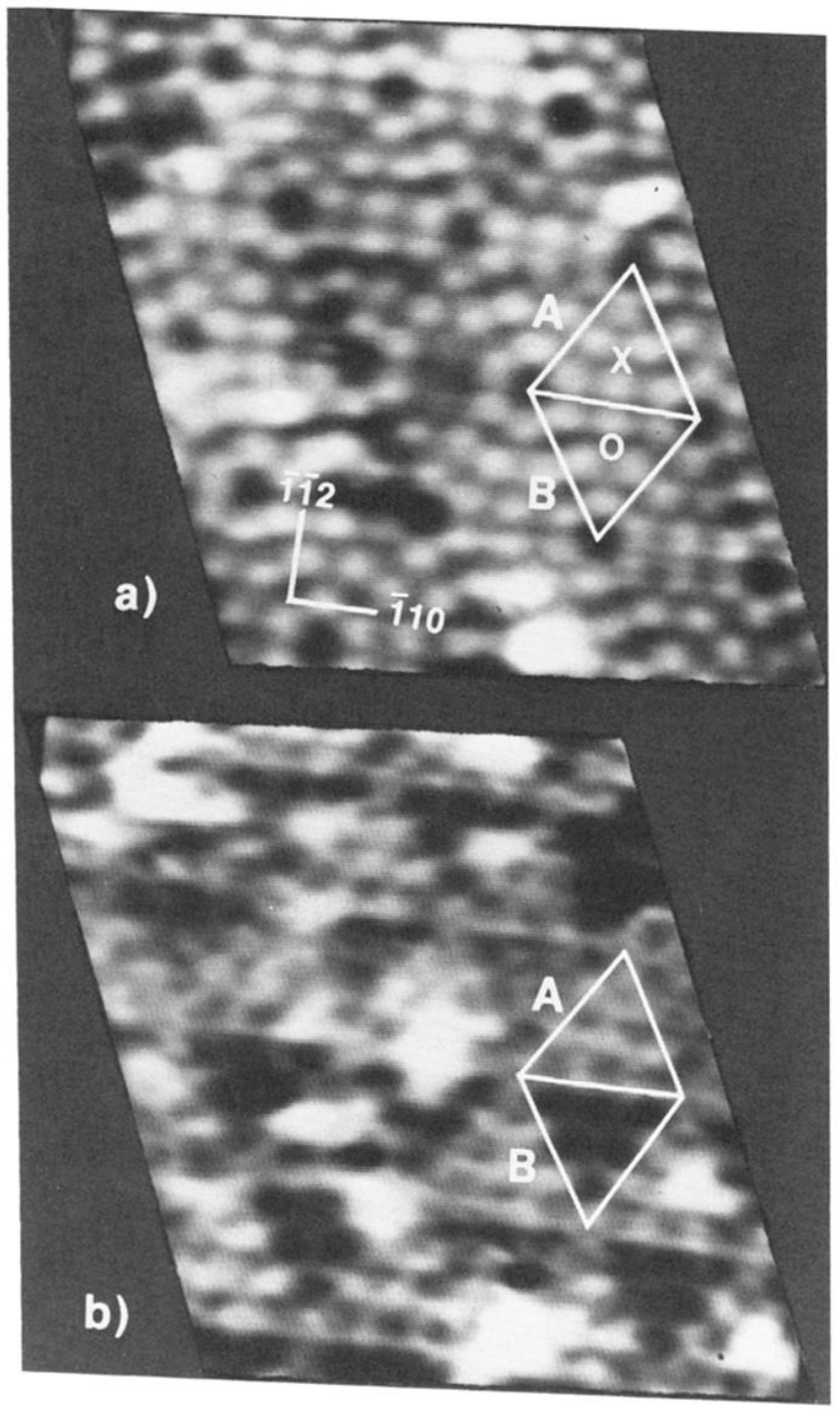

FIG. 1. (a) Gray-scale tunneling image of the $\operatorname{Si}(111) 7 \times 7$ surface used for the tunneling-field-emission studies. Light areas are high, while dark areas are low, with a total range of $1 \AA$. (b) Simultaneous conductance image with light representing increased signal and dark representing decreased signal (arbitrary units). The $7 \times 7$ unit mesh is depicted as a rhombus built up from two equilateral triangles. The surface orientation is as shown, with the A and B designating triangular subunits with vertices pointing in $\langle 2 \overline{11}\rangle$ and $\langle\overline{2} 11\rangle$ directions, respectively. The points $\mathrm{x}$ and $\mathrm{o}$ indicate the centers of the subunits. 